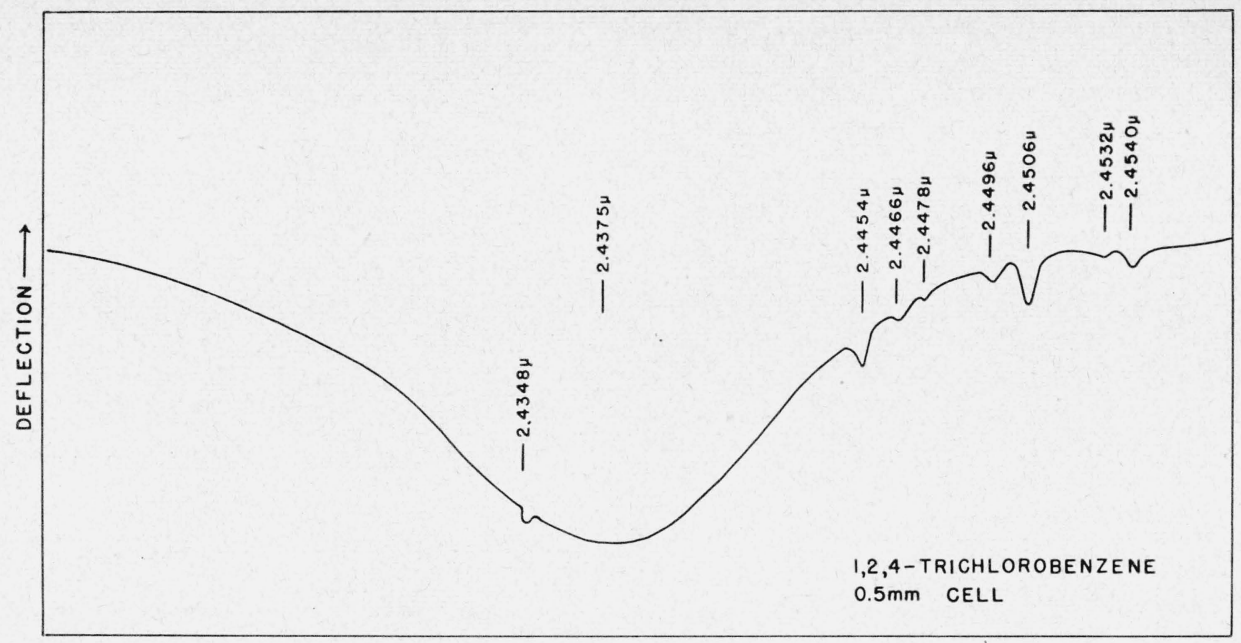

WAVELENGTH $\longrightarrow$

FiguRE 8. Absorption band at $2.4375 \mu$ of 1,2,4-trichlorobenzene with water vapor lines superimposed on the spectrum, as measured with the 15,000 lines-per-inch grating instrument.

Washington, August 29, 1950

\title{
High-Pressure Apparatus for Compressibility Studies and its Application to Measurements on Leather and Collagen
}

\author{
By Charles E. Weir
}

\begin{abstract}
The design and construction of apparatus to be used to measure volume changes of solids (or liquids) between 1,000- and 10,000-atmosphere pressure is described in detail. Calibration of the equipment and its use in determining the compression $\left(-\Delta V / V_{0}\right)$ of leather are discussed. The compression of leather between 1,000 and 10,000 atmospheres is reported. The compression between 2,000- and 10,000-atmosphere pressure is approximately 7 percent and appears to be relatively unaffected by moisture content, type of tannage, or sample variation. The compression of all leathers tested is described by the equation $-\Delta V / V_{0}=$ $1.23 \times 10^{-5}(P-2000)-5.60 \times 10^{-10}(P-2000)^{2}+1.35 \times 10^{-14}(P-2000)^{3}$.
\end{abstract}

\section{Introduction}

A Bureau program of measurement of physical constants of leather and collagen has resulted in a determination of the expansivity of leather and collagen [1]. ${ }^{1}$ These experiments also demonstrated that the shrinkage of leather, heretofore considered as analogous to a melting, was in reality a phenomenon occurring over a range of temperatures and resulted in an increase in real volume of the leatherwater system studied. Subsequent studies on the rate of shrinkage as a function of temperature, tannage, liquid medium, etc., have been reported $[2,3]$. In view of the results an investigation of the effect of pressure on leather and the shrinkage process was indicated, including a determination of the compressibility of leather for which only estimates have been available [4].

Experiments involving appreciable pressures are extremely exacting mechanically and have been

\footnotetext{
${ }_{1}$ Figures in brackets indicate the literature references at the end of this paper.
}

performed in few laboratories. To obtain information on the apparatus and techniques involved in such measurements, preliminary experiments were conducted on equipment that has been used for many years in the Geophysical Laboratory of the Carnegie Institution of Washington, for measurements at pressures as high as 12,000 bars. Subsequently similar equipment has been constructed at this Bureau and used in conjunction with a hydraulic press in the Geophysical Laboratory. This report deals with a description of the apparatus and a part of the data obtained.

\section{Apparatus}

In theory the apparatus required is simple; in practice, however, limitations imposed by the strength of materials require exacting machine work and the best of materials. An experiment consists essentially of forcing a piston into a vessel containing the sample under study immersed in a suitable liquid, and record- 
ing the depth of penetration of the piston at known internal pressures.

The apparatus required consists of-

1. A thick-walled pressure vessel having a smooth bore and a second opening provided to permit measurement of the internal hydrostatic pressure.

2. A leak-proof piston, or plug, which is forced into the bore.

3. A ram designed to drive the plug into the vessel.

4. A leak-proof plug carrying an electrically insulated lead from the internal pressure-gage to the external pressure-measuring equipment.

5 . The pressure-measuring equipment.

6. A hydraulic press to actuate the ram.

7. Assorted extractors to remove washers, etc.

The pressure vessel and accessories to be described are made in accordance with designs perfected by workers at the Geophysical Laboratory and are the result of wholehearted cooperation by these workers. The equipment is very similar to that described in considerable detail in 1919 by Adams, Williamson, and Johnston [5], but it is believed that a more recent highly detailed description of the apparatus, its fabrication, and use may be of interest and value to potential workers in this field.

The pressure vessel consists of a cylinder of SAE 4340 steel - a carbon, nickel, chrome, manganese, molybdenum, alloyed steel having "deep-hardening", properties -5 in. in diameter and $12 \mathrm{in.} \mathrm{long.} \mathrm{The}$ cylinder, partly shown in figure 1, contains an axial hole $5 / 8$ in. in diameter and 7 in. long. At the bottom of this bore a 2-in.-long hole is drilled, which decreases from $1 / 8$ to $1 / 16$ in. in diameter and serves to connect the pressure gage with the contents of the vessel. An opening for the pressure gage and its plug is made into the side of the vessel to connect with the bottom of the $1 / 16$-in. diameter axial hole. The gage opening consists of an outer hole $1 \frac{11}{4}$ in. deep designed to fit a bolt $1 \frac{1}{4} \mathrm{in}$. in diameter having 12 threads per inch; a central portion $3 / 4$ in. in diameter, 1 in. deep having a flat bottom that meets the wall at right angles; and an innermost portion $5 / 16$ in. in diameter extending slightly beyond and connecting with the axial $1 / 16^{-i n}$. diameter hole. After initial machining, this vessel was heat-treated to procure a hardness of Rockwell C-40. The scale produced in the heat treatment was removed, and the $5 / 8$-in. axial bore that was distorted was ground and lapped to produce a fine finish.

Before the pressure vessel could be used for highpressure work it had to be subjected to a pressure seasoning followed by a refinishing operation. After the initial machining, the bore of this vessel was observed to increase in diameter by several mils on application of pressure of approximately 10,000 atm. The vessel, however, can be rendered stable, at least in this pressure range, by a seasoning process consisting of subjecting the vessel to an internal pressure far in excess of any subsequently contemplated pressure [6]. For this seasoning, special plugs, which will be described later, were used to fill the openings in the vessel. In seasoning, pressure was applied in increments of 2,000 atm, pressure being maintained for 30

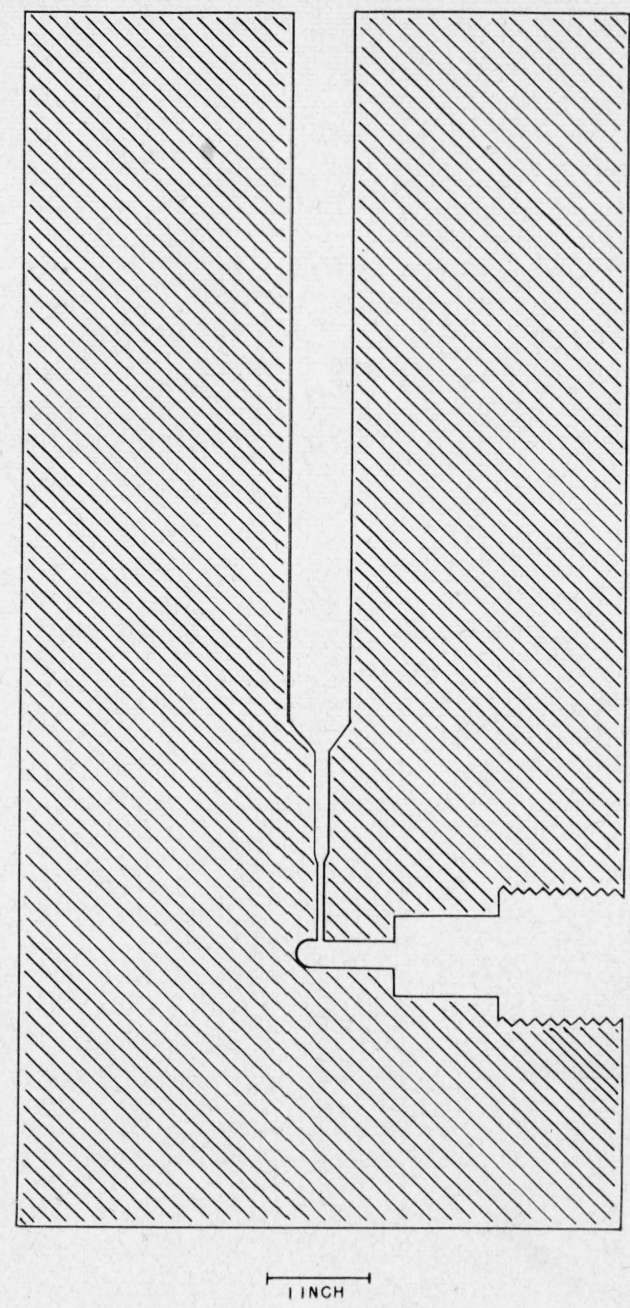

Figure 1. Section of pressure vessel.

min after each increment, until a maximum calculated internal pressure of $23,000 \mathrm{~atm}$ was attained. At the conclusion of this operation all fittings were found to be damaged, while the ressel had increased noticeably in diameter, externally as well as internally. In the refinishing operation it was necessary to enlarge the bore to $0.696 \mathrm{in}$. compared to the initial value of $0.630 \mathrm{in}$. The effectiveness of the seasoning treatment is shown by the fact that the vessel has been in use for several months, withstanding approximately 100 applications of pressures as high as 10,200 atm, with no measurable increase in diameter of the bore.

The movable leak-proof plugs [6] and associated washers are shown in figure 2. Plug $\mathrm{A}$ is the type used during seasoning and is designed so that the washers may undergo large lateral expansions to follow the considerable distortion occurring in seasoning. Plug B is used in compressibility measurements. Both plugs are made of Stentor steel, an oil-quenching manganese steel, and consist essentially of a body and stem each $3 / 4$ in. in length when finished. The stem is $5 / 16$ in. in diameter, and is threaded for the upper $3 / 8$ in. of its length with 24 threads per inch to 


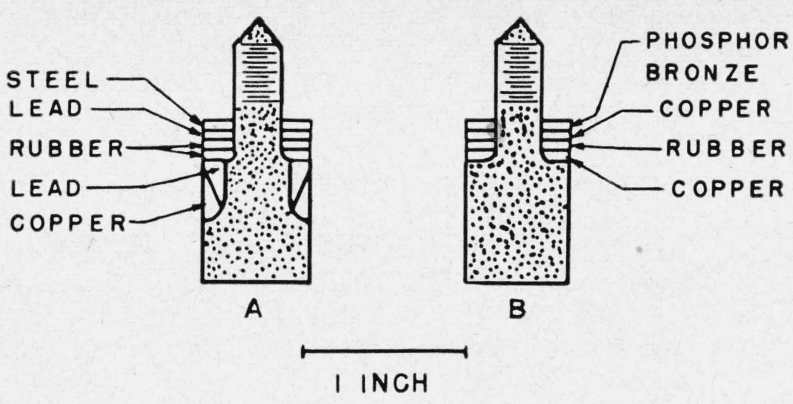

Figure 2. Sections of leak-proof plugs and washers.

permit extraction. It is very important that the junction of stem and body be filletted to prevent "pinching off" of the stem by the washers. In fabrication, the plug is machined to within 25 mils of the desired size, and heat-treated to procure extreme hardness (at least Rockwell C-60). The hardened piece is ground to size and polished to produce a fine finish, which is designed to minimize bigh stresses set up in grooves existing in the ground surface. The body of the plug is finished to be $1 / 2$ to 1 mil smaller than the bore of the vessel. Best results have been obtained with the smaller clearance of $1 / 2$ mil.

After compression measurements, the frictional force between the washers and the bore is of the order of 800 atms, and the plug must be extracted forcibly. In extraction, large stresses are set up in the stem of the plug, and difficulties have been encountered through breaking of the hardened stem. The failures appeared principally when the stem had been threaded prior to hardening, and were probably the result of small cracks in the threads formed in quenching. The best procedure has been found to grind the threads on the hardened stem. No failures to date have occurred in plugs made in this manner. It might be advantageous to draw the stem somewhat after finishing, but the necessity of resorting to this treatment has not been demonstrated.

The ram, used to drive the movable plug is shown in figure 3, and consists of a cold-rolled steel head shrunk onto a glass-hard Stentor steel shaft. The head serves to center the ram in the press and is relatively unimportant, since all stresses are borne by the shaft. The rod contains a recess at its lower end, which is finished to provide ample clearance of the stem of the plug that fits into it. Considerable clearance is advisable to prevent snapping the stem if moderate tilting occurs. The diameter of the shaft must be such that lateral swelling under compression will not cause binding in the bore of the vessel. In this instance the shaft is 5 mils smaller than the bore. Barring explosions, the ram travel is seldom greater than 2 in., and the length of the shaft should be kept to a minimum to prevent failure from buckling.

The pressure-gage plug, the insulated electrical lead, and its associated washers are shown in figure 4 . This is the most complex fitting required and has caused more failures (including explosions) than all

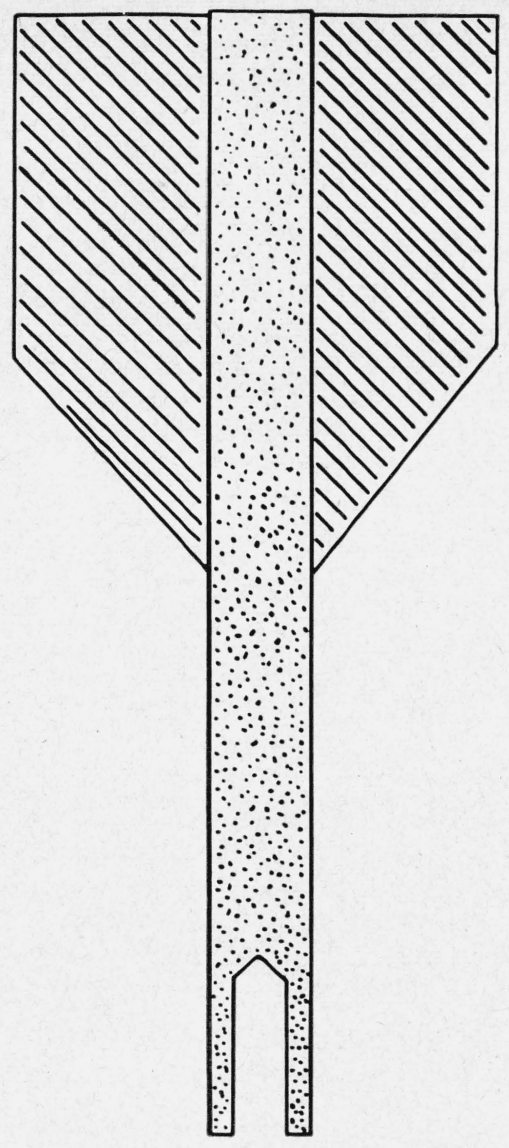

\section{$1 \mathrm{INCH}$}

Figure 3. Section of ram.

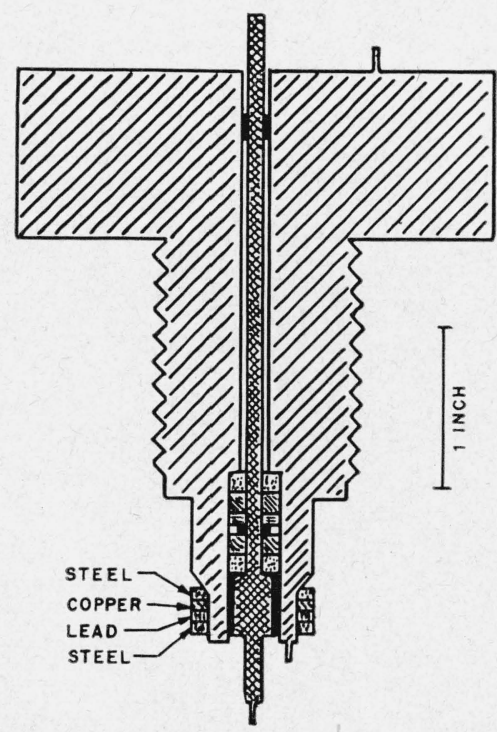

Figure 4. Section of gage plug and insulated lead. 
others combined. The plug and lead are both made of SAE 3440 steel hardened to Rockwell C-40.

The electrical lead consists of a rod $4 \% 3 \mathrm{in}$. long, being $5 / 32$ in. in diameter for $3 \frac{1 / 2}{\mathrm{in}}$. of its length, having a shoulder $0.262 \mathrm{in}$. in diameter and $3 / 8 \mathrm{in}$. long, and ending in another section $5 / 32$ in. in diameter and $1 / 2$ in. long. The tip of this latter section is approximately $1 / 32$ in. in diameter to facilitate silversoldering to the gage coil.

The gage plug is $1 \frac{1}{4} \mathrm{in}$. in diameter and is threaded for $1 \frac{1}{2} \mathrm{in}$. with 12 threads per inch. The end of the bolt is machined to two concentric cylindrical surfaces, respectively, $3 / 4$ in. in diameter and $1 / 2$ in. long, and $0.615 \mathrm{in}$. in diameter and $1 / 4 \mathrm{in}$. long. These surfaces are connected with a conical frustum $1 / 8$ in. long, which produces a seal with the packing washers. $A$ recess 1 in. deep and $5 / 16$ in. in diameter, having a flat bottom with no fillet at its wall, is machined in the end of the bolt, and a $7 / 32-i n$. diameter hole penetrates the remaining portion of the bolt. A $1 / 4$-in.-deep hole $1 / 32$ in. in diameter is drilled in each face of the bolt, and an oversized pin is driven into each hole to serve as a convenient point for securing electric contact.

The insulating washers shown around the electrical lead are in order from the bottom of the cavity: 1/8-in. limestone, 5/16-in. outside diameter, $5 / 32$-in. inside diameter; $1 / 8$-in. soapstone, $5 / 16$-in outside diameter, $5 / 32$-in. inside diameter; $1 / 8$-in. Neoprene rubber, 0.317 -in. outside diameter, $5 / 32$-in. inside diameter; $1 / 32$-in. steel, $1 / 4$-in. outside diameter, $5 / 32$-in. inside diameter; $1 / 8$-in. soapstone and $1 / 8$-in. limestone as before. This assembly is seated by using a small jig and an arbor press.

Two Bakelite washers not associated with the pressure seal are useful in preventing short circuits of the electrical lead, which may arise from tilting of this lead under pressure. One washer, $7 / 32$-in. outside diameter and $5 / 32$-in. inside diameter, serves to center the shaft on the low-pressure side, while the other, a thin shell 0.312 -in. outside diameter and $0.262-\mathrm{in}$. inside diameter, protects the shoulder from the plug on the high-pressure side.

In seasoning, a two-piece fitting was used, one being a solid hardened Stentor steel plug having the shape of the end of the plug, the other a $1 \frac{1 / 4}{4}$-in. bolt to hold the plug in place.

In operation the worker is protected by placing shields around the plug. Although no tendency has appeared toward failure of the threads, even at seasoning pressures, several failures have occurred in which the electrical lead was ejected with high velocity. These failures were all traced to errors in construction or to failure of steel parts. No difficulties, aside from electric short circuits, have been experienced with the arrangement described. The short circuits have resulted from slow extrusion of the limestone insulating washers into the pressure vessel, and use of a fine-grained lithographic limestone apparently has eliminated this difficulty.

The pressure gage consists of a coil of No. 38DSC manganin wire having a resistance of approximately $100 \mathrm{ohms}$. It is wound in two layers on thin paper, shellacked, and baked for $15 \mathrm{hrs}$ at $125^{\circ} \mathrm{C}$. Half-inch lengths of platinum wire are silver-soldered to the ends of the coil and in turn to the insulated electrical lead and the pin in the face of the plug. The gage coil is mounted on the end of the electrical lead, which fits through the coil and provides support.

The properties and seasoning of manganin pressure gages are discussed in detail by Adams, Goranson, and Gibson [7]. Gages used in these experiments were calibrated by using the water-ice VI transition, which occurs at $8,710 \mathrm{~atm}$ at $20^{\circ} \mathrm{C}$ [8]. As the pressure coefficient of manganin is essentially linear [9], this one point plus the known resistance at $1 \mathrm{~atm}$ provides a calibration sufficiently accurate for the present purpose.

The freezing-point apparatus consists of a tube containing distilled water and a few particles of ground glass to facilitate crystallization. The neck of the tube is drawn to a fine capillary, and the tube is inverted and slipped into a steel tube containing several milliliters of mercury. The mercury serves to seal the tube and permit transfer of pressure to the enclosed water. Calibration is performed by subjecting the freezing-point apparatus to pressure in excess of the expected freezing pressure, freezing being noted as a rapid and sudden decrease in resistance of the gage coil. The pressure is then lowered until a moderate rate of rise of resistance corresponding to melting of ice VI is noted. After a period of approximately $1 \mathrm{hr}$, an equilibrium pressure is attained, but extreme care must be exercised to insure coexistence of water and ice VI at this point. Generally, several equilibrium measurements are required on freezing as well as melting, and such measurements should be in good agreement.

The electric equipment used for measuring the resistance of the gage coil was a $\mathrm{G}-1$ Mueller Bridge. This is an unthermostatted Wheatstone bridge of the shunted-decade type, having a range 0 to $51.111 \mathrm{ohms}$ available in steps of $0.0001 \mathrm{ohm}$. The method of measurement differs from that used by other principal workers in this field $[5,7]$ and was suggested by E. F. Mueller of this Bureau.

As the pressure gage is approximately a $100-\mathrm{ohm}$ coil, the bridge cannot be used directly to measure the resistance of the coil. Two similar gage coils are used, with one coil-preferably the smaller of the two-being connected directly into one of the arms of the bridge where it serves essentially as a comparison resistor. This coil is immersed in the same liquid as is used for pressure transmission, and is mounted in a well in a large metal block attached to the side of the pressure vessel. The second coil is the pressure coil and is mounted inside the vessel, as described previously. One battery lead to the bridge is made at the junction of the two coils, as shown in the circuit diagram of the electric set-up in figure 5. In operation the bridge measures the difference in resistance of the two gage coils, one of which is subjected to changes in pressure and temperature - ambient temperature - whereas the other is subjected only to similar temperature changes. As the resistance of manganin increases with pres- 


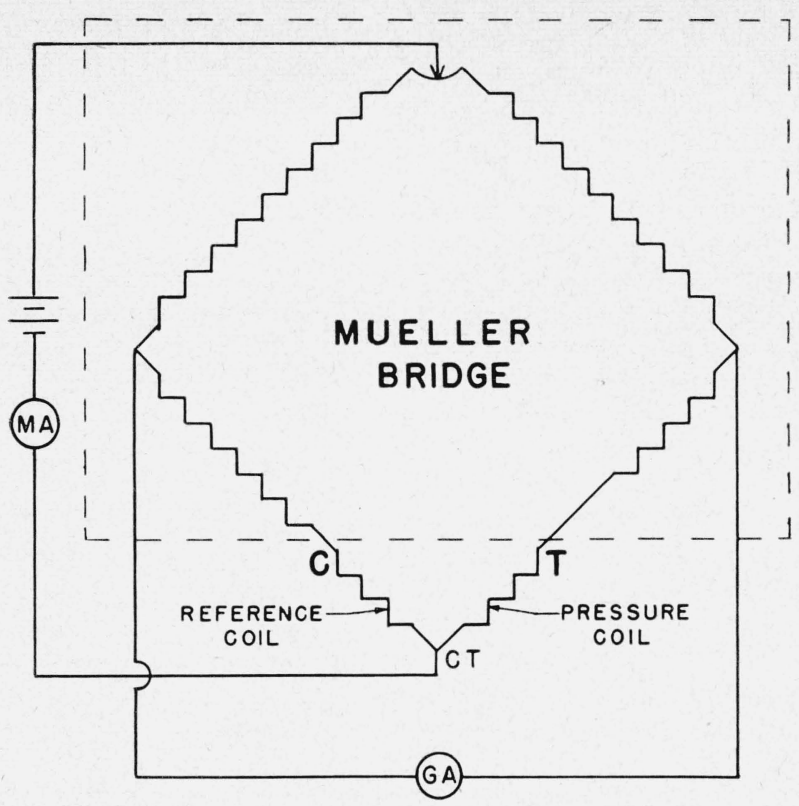

FIgURE 5. Schematic diagram of pressure-measuring equipment.

sure, the pressure coil should have the slightly larger resistance to eliminate the necessity for passing through a zero resistance reading. A commutator and mercury contact switch were included in the circuit but were not used in these experiments.

When two $1 \frac{11}{2}-\mathrm{v}$ dry cells were used, the total bridge current was $12 \mathrm{ma}$, and the gage coil current was $6 \mathrm{ma}$. With this current a deflection of $1 / 3 \mathrm{~mm}$ for each 0.0001-ohm step was obtained on the galvanometer scale set up at a distance of $2 \mathrm{~m}$. An atmosphere was found to be equivalent to approximately $0.00026 \mathrm{ohm}$, and the sensitivity was therefore such that a pressure of less than 1 atm could be read directly.

The maximum variation in resistance of the gage coils noted at $1 \mathrm{~atm}$ was equivalent to $\pm 3 \mathrm{~atm}$, and might be caused by the effect of temperaturea maximum temperature variation of $\pm 2^{\circ} \mathrm{C}$ occurred during all measurements on the bridge or on the coils or by the effect of pressure on the gage coil resistance. In the absence of precise temperature control this variation was ignored, as it represented a negligible source of error. No difficulties due to contact resistances in the switches were ever experienced.

No description of the accessories required will be given. A complete set of extractors for every part is required, but the design of such instruments is arbitrary. The extractor generally takes the form of a shaft threaded externally at one end and either externally or internally at the other. A large nut fitting the former end is screwed down against a convenient bearing surface and serves to extract the desired part, which is screwed into the latter end of the shaft. It is of the utmost importance that suitable provision be made for extracting every piece before pressure is imposed, for even though parts may have large clearances they are often found to be solidly joined after a single application of pressure.

The dial gage used to measure piston travel was attached to the top platen of the hydraulic press. This gage had a useful range of 0.400 in. and was graduated in 0.0001 in. Suitable gage blocks were used to extend the range of the gage. Calibration showed a maximum error of \pm 0.0001 in., and no corrections were applied to the readings.

A press suitable for the measurements was not available at this Bureau, and through the cooperation of the Geophysical Laboratory of the Carnegie Institution of Washington the equipment was set up for use with the 500-ton, hand-operated hydraulic press previously described [5].

\section{Experimental Method and Calculations}

Measurements consist of readings of the depth of the plug in the vessel - as denoted by the dial gageand the corresponding pressure [5]. Observations are made in order from the maximum pressure at every $1,000 \mathrm{~atm}$. To insure uniform behavior of the packing washers, all measurements are made with the plug moving into the vessel. This situation is obtained by decreasing the pressure to $50 \mathrm{~atm}$ below the desired value, and, after a pause to permit attainment of thermal equilibrium, pumping back as closely as possible to the desired pressure. The dial gage readings obtained are interpolated to even thousands of atmospheres to simplify the calculation. This procedure is repeated until the "reference pressure," here 2,000 atm, is reached. Occasionally observations are made at 1,000 atm also, but frictional forces often render these values unreliable. A similar measurement is performed with the specimen replaced by a steel bar having similar volume. The two sets of data are used to calculate the compression of the specimen in terms of that of the steel bar, which is known [10].

The equation required for the calculation was given by Adams et al. [5], but the terminology used here is that given by Adams [13]. The equation is as follows

$$
\begin{aligned}
k-k^{\prime}= & S / V_{0}\left\{\Delta L(1+\alpha P)+\Delta l^{\prime} \alpha\left(P-P^{\prime}\right)+\right. \\
& {\left.\left[\Delta l^{\prime}\left(1+\alpha P_{0}\right)+\frac{V^{\prime}-V_{r}^{\prime}}{S}\right]\left(\frac{k_{l}-k_{l}^{\prime}}{1-k_{l}^{\prime}}\right)\right\}+ } \\
& \left(k_{r}-k_{r}^{\prime}\right) \frac{\left(V_{r}\right)_{0}}{V_{0}}
\end{aligned}
$$

In this equation $P$ refers to pressure in atmospheres and $V$ volume; subscripts zero denote $1 \mathrm{~atm}$, subscripts $r$ and $l$ refer to steel reference bar and confining liquid, respectively, and primes denote the experimental reference pressure of $2,000 \mathrm{~atm} ; k$ is the compression $\left(V-V_{0}\right) / V_{0}=-\Delta V / V_{0}$ at pressure $P ; k^{\prime}$ is the compression at $2,000 \mathrm{~atm}\left(V^{\prime}-V_{0}\right) / V_{0}$; 
TABLE 1. Correction terms used in calculations of compressibility

\begin{tabular}{|c|c|c|c|c|}
\hline & & & \\
& $\left(k_{r}-k_{r}^{\prime}\right)$ & $\frac{k_{l}-k_{l}^{\prime}}{1-k_{l}}($ Varsol $)$ & $\alpha P$ & $\alpha\left(P-P^{\prime}\right)$ \\
\hline$a t m$ & & & & \\
\hline 10,000 & & & & \\
9,000 & .00472 & 0.1383 & 0.0130 & 0.0104 \\
8,000 & .00414 & .1279 & .0117 & .0091 \\
7,000 & .00352 & .1169 & .0104 & .0078 \\
6,000 & .00296 & .1037 & .0091 & .0065 \\
5,000 & .00237 & .0893 & .0078 & .0052 \\
4,000 & .00178 & .0730 & .0065 & .0039 \\
3,000 & .00060 & .0536 & .0052 & .0026 \\
2,000 & .00000 & .0295 & .0039 & .0013 \\
1,000 & -.00060 & -.0418 & .0026 & .0000 \\
& & & .0013 & -.0013 \\
\hline
\end{tabular}

$S$ is the cross-sectional area of the bore at $1 \mathrm{~atm}$ $\left(245.88 \mathrm{~mm}^{2}\right) ; \alpha$ is the pressure coefficient of this area $\left(1 / A_{0}\right)(d A / d P)=1.3 \times 10^{-6} / \mathrm{atm} ; \Delta L$ is the difference in travel of the piston at pressure $P$ reckoned from the reference pressure of 2,000 atm in the measurements on steel bar and specimen; and $\Delta l^{\prime}$ is the absolute difference in the position of the piston at the reference pressure in the runs on reference steel and specimen.

It is clear that if $V^{\prime}=V_{r}^{\prime}$ and if $\Delta l^{\prime}=0$, three terms on the right side of eq 1 disappear. These terms then may be considered as correction terms arising from inequality of volumes of reference steel and specimen and differences in volume of confining liquid. The contribution of these terms may be made negligible if suitable care is exercised in selecting the volume of the reference steel and in filling the pressure vessel.

The values of the correction terms of the right side of eq 1 are given in table 1 . The value of $\alpha$ was obtained from application of the equations given by the theory of elasticity for deformation of a cylinder under internal pressure [11]. The compression of the steel reference bar follows from the compressibility of steel given by Bridgman [10] converted into atmospheres. Compression values for Varsol were determined experimentally on the Varsol actually used, and were calculated by setting $V_{0}=V^{\prime}=0$ in eq 1 .

The volumes of the specimens studied are measured by the method of hydrostatic weighing at atmospheric pressure. The materials of interest have large compressibilities, which are functions of pressure, and the values of the volumes at 2,000 atm required in eq 1 are not easily obtained by successive approximations involving successive calculations and extrapolation to the known values at $1 \mathrm{~atm}$. To place the determination of values of $V^{\prime}$ on a mathematical basis, eq 1 was rearranged in the form

$$
\begin{aligned}
-\frac{\Delta V}{V_{0}}= & \frac{V^{\prime}}{V_{0}}\left[\left(\frac{k_{l}-k_{l}^{\prime}}{1-k_{l}^{\prime}}\right)-1\right]+S / V_{0}[(1+\alpha P) \Delta L+ \\
& \left.\alpha\left(P-P^{\prime}\right) \Delta l^{\prime}+\left(\Delta l^{\prime}-\frac{V_{r}^{\prime}}{S}\right)\left(\frac{k_{l}-k_{l}^{\prime}}{1-k_{l}^{\prime}}\right)\right]+ \\
& 1+\left(k_{r}-k_{r}^{\prime}\right) \frac{\left(V_{r}\right)_{0}}{V_{0}}
\end{aligned}
$$

All quantities on the right side of eq 2 are known explicitly except $V^{\prime}$.

The compression, $-\left(\Delta V / V_{0}\right)$, has been found to be well represented by a cubic in pressure, as given by

$$
-\frac{\Delta V}{V_{0}}=a P+b P^{2}+c P^{3} .
$$

The right side of eq 2 and 3 are equated, and the value of $V^{\prime} / V_{0}$ was obtained from the data by the method of least squares. The value of $V^{\prime}$ so obtained was used in equation 1 to calculate experimental values of $k-k^{\prime}$.

\section{Preparation and Treatment of Speci- mens}

Measurements were made on three types of commercial leather: chrome, retan, and vegetable tanned, as well as two types of collagen: hide collagen and kangaroo tail tendon.

Test samples of leather and hide collagen consisted of a stack of disks $5 / 8$ in. in diameter and 4 in. long, held together by means of fine wire. The sample of tendon was a bundle of tendons of similar dimensions. The disks comprising the sample were subjected to the following treatments prior to test.

Two samples of chrome tanned hydraulic packing leather denoted as $\mathrm{A}$ and $\mathrm{B}$, cut from different areas of the same hide were tested. The specimens were degreased for $24 \mathrm{hr}$ with chloroform, washed in running water for $24 \mathrm{hr}$, and conditioned at $72^{\circ} \mathrm{F}$ and 50-percent relative humidity for 2 mo prior to use.

After measurements on these conditioned samples, the Varsol was removed by evaporation at room temperature for 1 week, and exposure to a temperature of $60^{\circ} \mathrm{C}$ for $24 \mathrm{hr}$. The samples were then dried at $100^{\circ} \mathrm{C}$ for $18 \mathrm{hr}$. Sample A was then tested and the Varsol removed again, as described previously. Both samples were then redried at $100^{\circ} \mathrm{C}$ for $24 \mathrm{hr}$ in vacuum $(<2 \mathrm{mmHg})$ and retested.

One sample of retan upper leather was degreased, washed, and conditioned as described previously. Tests were made only on the conditioned sample.

Two dried samples of vegetable tanned leather obtained from neighboring areas of the same hide were tested. One sample, denoted as A, was prepared by degreasing, washing, and drying at $100^{\circ} \mathrm{C}$, as described. The second sample, B, was washed, dried in vacuum for $16 \mathrm{hr}$, degreased, and then dried at $100^{\circ} \mathrm{C}$.

Partially purified hide collagen was obtained by dehydration of a freshly flayed cowhide with acetone. The dehydrated material was split to remove the grain and flesh layers, and the resulting corium was conditioned and used for test.

Tendons were conditioned prior to test and received no chemical treatment.

All samples were weighed prior to test and were then subjected to impregnation with Varsol under a vacuum of approximately $20 \mathrm{~mm} \mathrm{Hg}$. Following compression determinations the samples were reweighed in Varsol. From the loss in weight in 
TABLE 2. Compressibility of conditioned chrome-tanned leather

\begin{tabular}{|c|c|c|c|c|c|c|c|c|}
\hline \multirow{3}{*}{ Pressure } & \multicolumn{5}{|c|}{$\begin{array}{c}\text { Sample A } \\
V_{0}=9.595 \mathrm{ml} ; V^{\prime}=9.333 \mathrm{ml} \\
\rho_{22}{ }^{\circ} \mathrm{C}=1.367_{0} \mathrm{~g} / \mathrm{cm}^{3}\end{array}$} & \multirow{2}{*}{\multicolumn{3}{|c|}{$\begin{array}{c}\text { Sample B } \\
\begin{array}{c}V_{0}=9.520 \mathrm{ml} ; V^{\prime}=9.281 \mathrm{ml} \\
\rho_{22}{ }^{\circ} \mathrm{C}\end{array}=1.362_{\gamma} \mathrm{g} / \mathrm{cm}^{3} \\
\text { Run- }\end{array}$}} \\
\hline & \multicolumn{5}{|c|}{ Run- } & & & \\
\hline & $\begin{array}{c}1 \\
\text { a } k-k^{\prime}\end{array}$ & $\begin{array}{c}2 \\
k-k^{\prime}\end{array}$ & $\begin{array}{c}3 \\
k-k^{\prime}\end{array}$ & $\begin{array}{c}4 \\
k-k^{\prime}\end{array}$ & $\begin{array}{c}\text { Average } \\
k-k^{\prime}\end{array}$ & $\begin{array}{c}1 \\
k-k^{\prime}\end{array}$ & $\begin{array}{c}2 \\
k-k^{\prime}\end{array}$ & $\begin{array}{c}\text { Average } \\
k-k^{\prime}\end{array}$ \\
\hline $\begin{array}{l}\text { atm } \\
10,000 \\
9,000 \\
8,000 \\
7,000 \\
6,000 \\
5,000 \\
4,000 \\
3,000 \\
2,000\end{array}$ & $\begin{array}{r}0.0693 \\
.0630 \\
.0558 \\
.0481 \\
.0398 \\
\\
.0319 \\
.0221 \\
.0121 \\
.0000\end{array}$ & $\begin{array}{r}0.0701 \\
.0641 \\
.0574 \\
.0495 \\
.0419 \\
\\
.0329 \\
.0222 \\
.0120 \\
.0000\end{array}$ & $\begin{array}{r}0.0674 \\
.0618 \\
.0553 \\
.0487 \\
.0399 \\
\\
.0310 \\
.0232 \\
.0120 \\
.0000\end{array}$ & $\begin{array}{r}0.0699 \\
.0643 \\
.0577 \\
.0493 \\
.0413 \\
\\
.0327 \\
.0226 \\
.0121 \\
.0000\end{array}$ & $\begin{array}{r}0.0692 \\
.0633 \\
.0565 \\
.0492 \\
.0407 \\
\\
.0321 \\
.0225 \\
.0120 \\
.0000\end{array}$ & $\begin{array}{r}0.0697 \\
.0644 \\
.0578 \\
.0510 \\
.0424 \\
\\
.0336 \\
.0236 \\
.0121 \\
.0000\end{array}$ & $\begin{array}{r}0.0702 \\
.0642 \\
.0573 \\
.0500 \\
.0418 \\
\\
.0345 \\
.0230 \\
.0125 \\
.0000\end{array}$ & $\begin{array}{r}0.0700 \\
.0643 \\
.0576 \\
.0505 \\
.0421 \\
\\
.0340 \\
.0233 \\
.0123 \\
.0000\end{array}$ \\
\hline & & rate valu & & & & & rnate va & \\
\hline $\begin{array}{l}10,000 \\
9,000 \\
8,000 \\
6,000\end{array}$ & $\begin{array}{r}0.0688 \\
.0632 \\
.0565 \\
.0492 \\
.0406\end{array}$ & $\begin{array}{r}0.0693 \\
.0630 \\
.0564 \\
.0482 \\
.0403\end{array}$ & $\begin{array}{r}0.0703 \\
.0632 \\
.0565 \\
.0492 \\
.0406\end{array}$ & $\begin{array}{r}0.0682 \\
.0628 \\
.0559 \\
.0488 \\
.0405\end{array}$ & $\begin{array}{r}0.0692 \\
.0630 \\
.0563 \\
.0489 \\
.0405\end{array}$ & $\begin{array}{r}0.0713 \\
.0658 \\
.0590 \\
.0513 \\
.0432\end{array}$ & $\begin{array}{r}0.0683 \\
.0628 \\
.0563 \\
.0496 \\
.0410\end{array}$ & $\begin{array}{r}0.0698 \\
.0643 \\
.0577 \\
.0504 \\
.0421\end{array}$ \\
\hline $\begin{array}{l}5,000 \\
4,000 \\
3,000 \\
2,000\end{array}$ & $\begin{array}{l}.0321 \\
.0225 \\
.0121 \\
.0000\end{array}$ & $\begin{array}{l}.0317 \\
.0218 \\
.0120 \\
.0000\end{array}$ & $\begin{array}{l}.0321 \\
.0225 \\
.0121 \\
.0000\end{array}$ & $\begin{array}{l}.0315 \\
.0223 \\
.0120 \\
.0000\end{array}$ & $\begin{array}{l}.0318 \\
.0223 \\
.0120 \\
.0000\end{array}$ & $\begin{array}{l}.0347 \\
.0239 \\
.0122 \\
.0000\end{array}$ & $\begin{array}{l}.0322 \\
.0227 \\
.0124 \\
.0000\end{array}$ & $\begin{array}{l}.0334 \\
.0233 \\
.0123 \\
.0000\end{array}$ \\
\hline
\end{tabular}

a $k-k^{\prime}$ is equivalent to the relative volume change, $-\Delta V / V_{0}$, reckoned from $2,000 \mathrm{~atm}$.

Varsol and the density of the Varsol, the volume of the samples was obtained. The density of Varsol was obtained by successive weighings of a steel bar in air, water, and Varsol, respectively, and was found to be $0.7811 \mathrm{~g} / \mathrm{ml}$.

\section{Results and Discussion}

\section{Reproducibility}

In order to evaluate the reproducibility of the measurements, duplicate experiments were made in many instances. A particular set of measurements was obtained in the following way.

An initial measurement was made on the steel reference bar, and was followed by a measurement on sample A and then on sample B. The second determinations were made by measuring sample $\mathrm{A}$, then sample B, and finally the reference bar. As only two measurements were made in 1 day these determinations required 3 days for completion. The values of compression could be calculated on the basis of either of the two measurements on the steel bar, and both calculations were performed. It is considered that the best calculation is that based on the run nearest in time to the reference run. Values based on the farthest removed reference run are given as alternate values. The results of this experiment are given in table 2 .

Runs 1 and 2 of the table refer to the experiment described, and two other runs performed at a different time on sample A are included for comparison. The agreement is good between runs, and within experi- mental error the compressions of samples A and B are not significantly different.

\section{Effect of Moisture on Compression}

Measurements were made on sample $A$ after drying it at $100^{\circ} \mathrm{C}$, and on both samples $\mathrm{A}$ and $\mathrm{B}$ after drying them at $100^{\circ} \mathrm{C}$ in vacuum, as described. The results are given in table 3 .

TABLE 3. Effect of moisture content on compressibility of chrome-tanned leather

\begin{tabular}{|c|c|c|c|c|}
\hline \multirow[t]{3}{*}{ Pressure } & \multicolumn{2}{|c|}{$\begin{array}{c}\text { Sample } \mathrm{A}^{\prime} \\
\text { Sample A dried } \\
\text { at } 100^{\circ} \mathrm{C} \\
V_{0}=7.882 \mathrm{ml} \\
V^{\prime}=7.608 \mathrm{ml} \\
\rho_{22^{\circ}} \mathrm{C}=1.3899 \mathrm{~g} / \mathrm{cm}^{3}\end{array}$} & \multirow{3}{*}{$\begin{array}{c}\text { Sample } \mathrm{A}^{\prime \prime} \\
\text { Sample } \mathrm{A}^{\prime} \text { dried } \\
\text { at } 100^{\circ} \mathrm{C} \text { in } \\
\text { vacuum } \\
V_{0}=7.691 \mathrm{ml} \\
V^{\prime}=7.481 \mathrm{ml} \\
\rho_{22^{\circ} \mathrm{C}}=1.3878 \mathrm{~g} / \mathrm{cm}^{3}\end{array}$} & \multirow{3}{*}{$\begin{array}{c}\text { Sample } \mathrm{B}^{\prime \prime} \\
\text { Sample B dried } \\
\text { at } 100^{\circ} \mathrm{C} \text { and } \\
\text { redried at } 100^{\circ} \mathrm{C} \\
\text { in vacuum } \\
V_{0}=7.617 \mathrm{ml} ; \\
V^{\prime}=7.409 \mathrm{ml} \\
\rho_{22}{ }^{\circ} \mathrm{C}=1.3866 \mathrm{~g} / \mathrm{cm}^{3} \\
k-k^{\prime}\end{array}$} \\
\hline & \multicolumn{2}{|c|}{ Run- } & & \\
\hline & $\begin{array}{c}1 \\
a k-k^{\prime}\end{array}$ & $\begin{array}{c}2 \\
k-k^{\prime}\end{array}$ & & \\
\hline $\begin{array}{l}\text { atm } \\
10,000 \\
9,000 \\
8,000 \\
7,000 \\
6,000 \\
5,000 \\
4,000 \\
3,000 \\
2,000 \\
1,000\end{array}$ & $\begin{array}{r}0.0683 \\
.0627 \\
.0570 \\
.0502 \\
.0414 \\
.0329 \\
.0240 \\
.0128 \\
.0000 \\
-.0119\end{array}$ & $\begin{array}{r}0.0687 \\
.0625 \\
.0568 \\
.0477 \\
.0413 \\
.0329 \\
.0235 \\
.0124 \\
.0000 \\
-.0118\end{array}$ & $\begin{array}{r}0.0706 \\
.0642 \\
.0572 \\
.0494 \\
.0417 \\
.0315 \\
.0229 \\
.0122 \\
.0000 \\
-.0134\end{array}$ & $\begin{array}{r}0.0710 \\
.0646 \\
.0571 \\
.0503 \\
.0414 \\
.0320 \\
.0231 \\
.0123 \\
.0000 \\
\end{array}$ \\
\hline
\end{tabular}

a $k-k^{\prime}$ is equivalent to the relative volume change, $-\Delta V / V_{0}$, reckoned from $2,000 \mathrm{~atm}$. 
It is surprising that the removal of moisture causes little or no change in compression of the leather. A total loss in weight of 18.6 percent is involved between conditioned samples of table 2 and the dry samples of table 3 . If it is assumed that this total loss in weight represents moisture [12], and the compression of a composite sample containing 18.6 percent of water and 81.4 percent of leather is calculated from the compressions of table 3 and known values for water [13], values are obtained that are of the order of 10 percent higher than the measured values of table 2 . Even if the moisture present in the leather is considered to be "bound water" under high pressure, it would appear that the compression of the leather should change upon removal of the water, except in the one case in which the compressibility of the combined water and the leather are nearly identical over the whole pressure range. The probability of such coincidence seems small. This finding deserves further experimental study on this and other hygroscopic materials containing varying amounts of moisture.

\section{Compressions of Other Types of Leather and Collagen}

The results of measurements of compressions of two types of collagen and retanned and vegetabletanned leather are given in table 4.

It is to be noted that the compression values of the vegetable-tanned samples agree but appear to be slightly lower than values obtained for chrometanned samples. Values for the retan leather, on the other hand, appear somewhat higher than values for chrome leather, while the values obtained for the collagens agree well both with each other and with the chrome leather. The divergences observed with vegetable and retanned leather may be real, but conclusions in this respect will not be made until further more extensive measurements are conducted.

\section{Compressibility of Leather}

To obtain an explicit expression for compression, a power series in pressure may be fitted to the experi- mental data. Terms of higher degree than the third in pressure have been found to contribute little to the efficacy of the fit, and a cubic equation in pressure appears to describe the data satisfactorily. Data obtained for different samples, tannages, and moisture contents might all be fitted with such equations, but since there are only slight differences in compressions of these materials the equations evolved would be expected to agree fairly well and to exhibit small variations in coefficients. Therefore, an equation of third degree in pressure was fitted only to the best data - the average values for sample $A$ in table 2 - which are considered as representative. Values obtained by use of this equation will closely approximate the experimental values obtained on all leathers tested. The equation obtained by use of the method of least squares is as follows

$$
\begin{gathered}
-\Delta V / V_{0}=1.23 \times 10^{-5}(P-2000)-5.60 \times 10^{-10}(P- \\
2000)^{2}+1.35 \times 10^{-14}(P-2000)^{3} .
\end{gathered}
$$

The compressibility obtained by differentiating the above equation is found to be

$$
\begin{aligned}
-\frac{1}{V_{0}} \frac{d V}{d P}= & 1.23 \times 10^{-5}-1.12 \times 10^{-9}(P-2000)+ \\
& 4.05 \times 10^{-14}(P-2000)^{2} .
\end{aligned}
$$

A previous estimate of the compressibility [4], $33 \times$ $10^{-6} / \mathrm{bar}$, is considerably larger than that given by the equation above. However, equation 2 shows the compressibility to be a function of pressure and the value of $33 \times 10^{-6}$ bar was obtained at an undetermined pressure much lower than the minimum pressure of 2,000 atm at which eq 2 applies. Extrapolation of eq 4 to atmospheric pressure yields a compressibility considerably smaller than $33 \times 10^{-6} / \mathrm{bar}$.

\section{Conclusions}

The design and construction of apparatus for measuring compression of solids (or liquids) in the pressure range 2,000 to $12,000 \mathrm{~atm}$ has been described. The results of measurements made on a natural high polymer, leather, show that the apparatus is capable of yielding data of good reproducibility.

TABLE 4. Compressibilities of collagen and leather

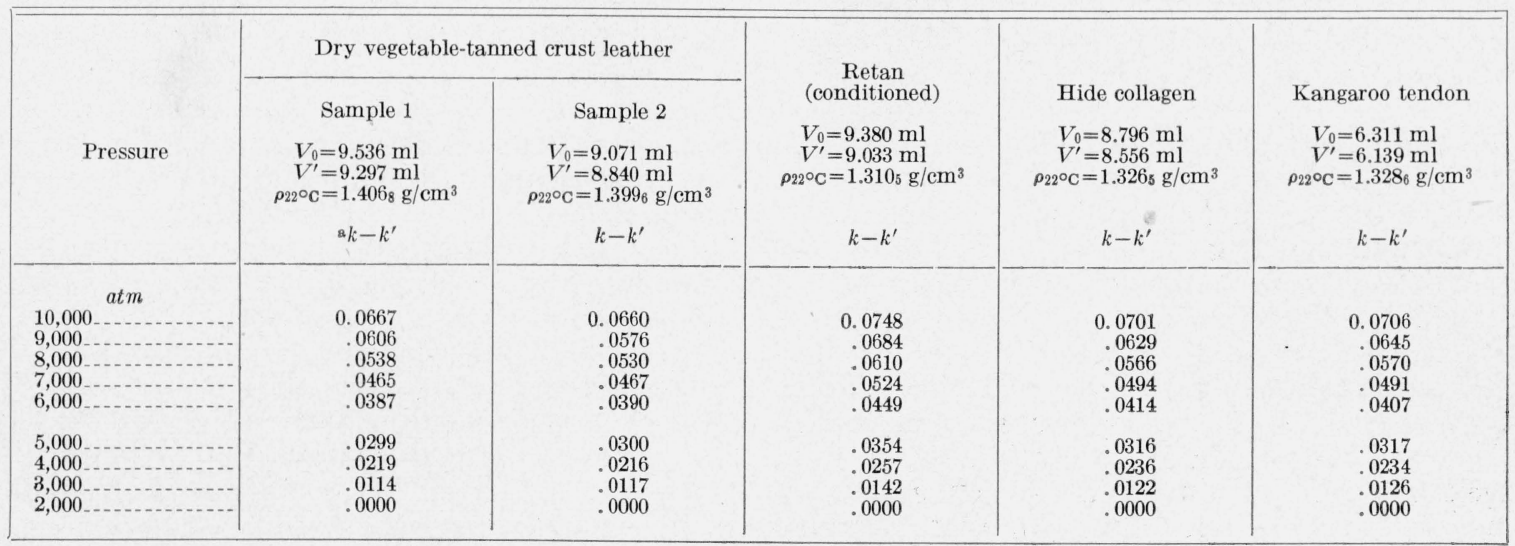

a $k-k^{\prime}$ is equivalent to the relative volume change, $-\Delta V / V_{0}$, reckoned from $2,000 \mathrm{~atm}$. 
The data obtained on leather are indicative of a compressibility that is intermediate between the compressibilities exhibited by most solids and those of liquids. The compression is not materially affected by moisture content. Values obtained for chrome-tanned leather and two types of collagen are in good agreement, but the compressions of vegetable and retanned leathers appear to be slightly different. Compressions of samples taken from different areas of the hide are in substantial agreement.

This investigation was made possible by the wholehearted cooperation of members of the Geophysical Laboratory of the Carnegie Institution of Washington and of the Bureau of Standards. The author is particularly indebted to L. H. Adams, H. S. Yoder, and J. Van den Huerk, of the Geophysical Laboratory, for encouragement, invaluable technical advice, and use of the facilities of their laboratory; and to E. F. Mueller and R. E. Wilson, of this Bureau, for advice and assistance in connection with the resistance measurements.

\section{References}

[1] C. E. Weir, J. Research NBS 41, 279 (1948) RP1924.

[2] C. E. Weir, J. Research NBS 42, 17 (1949) RP1947.

[3] C. E. Weir, J. Research NBS 44, 599 (1950) RP2106.

[4] C. E. Weir, J. Research NBS 35, 257 (1945) RP1672.

[5] L. H. Adams, E. D. Williamson, and J. Johnston, J. Am. Chem. Soc. 41, 12 (1919).

[6] P. W. Bridgman, Pro. Am. Acad. Arts \& Sci. 49, 627 (1914).

[7] L. H. Adams, R. W. Goranson, and R. E. Gibson, Rev. Sci. Instr. 8, 230 (1937)

[8] P. W. Bridgman, Pro. Am. Acad. Arts \& Sci. 47, 441 (1911).

[9] P. W. Bridgman, Pro. Am. Acad. Arts \& Sci. 47, 321 (1911).

[10] P. W. Bridgman, Pro. Am. Acad. Arts \& Sci. 74, 11 (1940).

[11] S. Timoshenko, Theory of elasticity (McGraw Hill Book Co., Inc., New York, N. Y. 1934)

[12] J. R. Kanagy, J. Am. Leather Chem. Assoc. 36, 609 (1941).

[13] L. H. Adams, J. Am. Chem. Soc. 53, 3769 (1931).

Washington, August 3, 1950.

\title{
Arc and Spark Spectra of Technetium
}

\author{
By William F. Meggers and Bourdon F. Scribner
}

\begin{abstract}
Four milligrams of highly purified technetium, loaned by the United States Atomic Energy Commission, were used to obtain a description of the arc and spark spectra characteristic of this fission product. Solutions containing 50 to 200 micrograms of technetium were dried on copper electrodes and excited by electric arcs or sparks. A stigmatic concave grating of 22-feet radius was employed to photograph the spectra from 2200 to 9000 angstroms, within which limits more than 2,300 lines characteristic of Tc atoms or ions were recorded. Wavelengths were measured relative to iron standards, relative intensities were estimated on a scale of 1 to 1000, and almost every line was definitely assigned either to neutral Tc atoms or to singly charged $\left(\mathrm{Tc}^{+}\right)$ions. The measured wavelengths range from 2261.30 to 8829.80 angstroms with average probable errors rarely exceeding \pm 0.01 angstrom. The average results of wavelength measurements and of intensity estimates for 2,121 lines in Te I and Te II spectra are presented. The strongest Te I lines have wavelengths $4297.06,4262.26,4238.19,4031.63$, and 3636.10 angstroms. The strongest Te II lines have wavelengths $2543.24,2610.00$, and 2647.02 angstroms. This description of Te I and Te II spectra will serve for spectro-chemical identification and for structural analyses of these spectra.
\end{abstract}

\section{Introduction}

In 1869, when the periodic chart of the atoms was first proposed, Mendeléeff $[1]^{1}$ saw that the chemical elements homologous to manganese were missing in the two succeeding periods; he called these ekamanganese and dwi-manganese. This was the first hint of the possible existence of the element under discussion. In the following half century many attempts, both chemical and spectroscopic, were made to detect these homologs of manganese, but without success. Moseley's [2] discovery in 1913 of a quantitative relationship between Roentgen frequencies and atomic numbers proved that atomic numbers $43,61,72,75,85$, and 87 were still unknown, and provided a new method of detection by means of Roentgen spectra. This method was applied in 1925 by Noddack, Tacke, and Berg [3], who reported

Figures in brackets indicate the literature references at the end of this paper. finding, in material extracted from columbite, three $K$ spectrum lines for atomic number 43 and five $L$ spectrum lines for atomic number 75 . These two atomic numbers are identical with the homologs of manganese, and the names masurium and rhenium were proposed for them. The discovery of rhenium was abundantly confirmed, and within a few years this new element became a commercial metal. In 1931 the arc spectrum of rhenium was described and interpreted by Meggers [4], who then inquired of the discoverers if a sample of masurium was available for spectroscopic investigation. The Noddacks ${ }^{2}$ replied that they had succeeded in concentrating some masurium, and that they would send the first milligram that could be spared. Unfortunately that milligram never materialized, and it later appeared that the extraction of element 43 from natural sources would be unlikely or even impossible [5].

\footnotetext{
${ }^{2}$ In a letter dated Dec. 22, 1931, from W. and I. Noddak to W. F. Meggers.
} 